ROTATION MEASUREMENT WITH THE USE OF A PULSED ER-DOPED FIBER LASER WITH INTEGRATED OPTIC CIRCUIT: PROPOSAL, SIMPLE THEORY, AND EXPERIMENT

\section{Chao-Xiang Shi}

Department of Electrical Engineering and Computer Science

University of Michigan

Beal Ave. 1301

Ann Arbor, Michigan 48109

\section{Received 25 February 1997}

ABSTRACT: We propose a novel pulsed fiber laser rotation sensor with a $\mathrm{LiNbO}_{3}$ integrated optic circuit, Sagnac fiber loop, Er-doped fiber, and semiconductor pumping lasers. A simple analysis is completed and the output equation is derived. The variance of the output pulse when rotation occurs is also analyzed. In addition, we also conducted a related experiment for rotation measurement. When the gain of the fiber amplifier is over the round-trip loss and the phase modulation frequency satisfies the total round-trip time, this optical system produces the laser emission in a pulse state. The rotation rate is measured from the shift of the peak position of the output pulse. It is shown that the resolution of rotation measurement increases if we use a smaller phase modulation depth. But the sharpness of the optical pulse must be maintained by reducing the losses (or increasing the gain of optical amplifier) in the whole optical system. (C) 1997 John Wiley \& Sons, Inc. Microwave Opt Technol Lett 15: 311-315, 1997.

Key words: Er-doped fiber; fiber laser; integrated optic circuit

\section{INTRODUCTION}

Fiber-optic rotation sensors (or fiber-optic gyroscopes) with the Sagnac effect for rotation measurement have been widely investigated for many years [1]. Basically, there are three kinds of fiber-optic gyroscopes. One is the interferometric fiber-optic gyroscope (I-FOG), which utilizes an optical interference between the $\mathrm{CW}$ (clockwise) and CCW (counterclockwise) waves that pass through a Sagnac loop [2]. A low-coherence light source (SLD or multimode LD) is used in the I-FOG, and the cost of whole gyro system is low. I-FOG has found many practical uses, such as in car navigation and many other industrial applications [3]. The second type of fiber gyroscope is the resonant fiber-optic gyroscope (R-FOG), which detects the change in resonant frequency that is due to the Sagnac effect in the high-finesse ring resonator [4]. The R-FOG uses a short fiber to conform the Sagnac loop and can obtain high resolution in rotation measurement. However, the R-FOG requires a highly coherent light source, which is expensive. Many worse phenomena, such as the Kerr effect and Backscattering effects must be considered [5]. The third type of FOG is an active fiber-optic gyroscope employing a stimulated Brillouin-scattering laser composed of a fiber-ring resonator (B-FOG). It translates the Sagnac phase shift into a beat frequency between the counterpropagation laser modes [6]. In this research we propose a novel fiber-optic rotation sensor with an integrated optic circuit, a Sagnac fiber loop, and an Er-doped fiber amplifier. This optical system produces a laser emission in the pulse state if the gain of fiber amplifier is greater than the whole round-trip transmission loss, and if the frequency of phase modulator and the total loop length satisfy a certain mode-lock condition. The measurement of rotation rate is based on the peak shift of output pulse induced by the Sagnac effect.

\section{BASIC CONFIGURATION AND EXPERIMENT SETUP}

The basic configuration and experimental setup are illustrated in Figure 1. The sensing part is composed of a Sagnac loop of elliptical-core polarization-maintaining fiber (about $164 \mathrm{~m}$ ), with an integrated optic circuit (from Ramer Inc. with insertion loss of about $10 \mathrm{~dB}$ at $1.55 \mu \mathrm{m}$ ), which contains a $\mathrm{LiNbO}_{3} \mathrm{Y}$-branch waveguide with metal electrodes for phase modulation. In addition, a polarizer is integrated within the optical IC. Therefore, this integrated optic circuit includes all the functions of fiber polarizer, fiber coupler, and phase modulator in conventional all-fiber I-FOG. In this work there is also a feedback loop (about $63 \mathrm{~m}$ ) supplying an optical amplification (about $30 \mathrm{~dB}$ at the small signal) with the

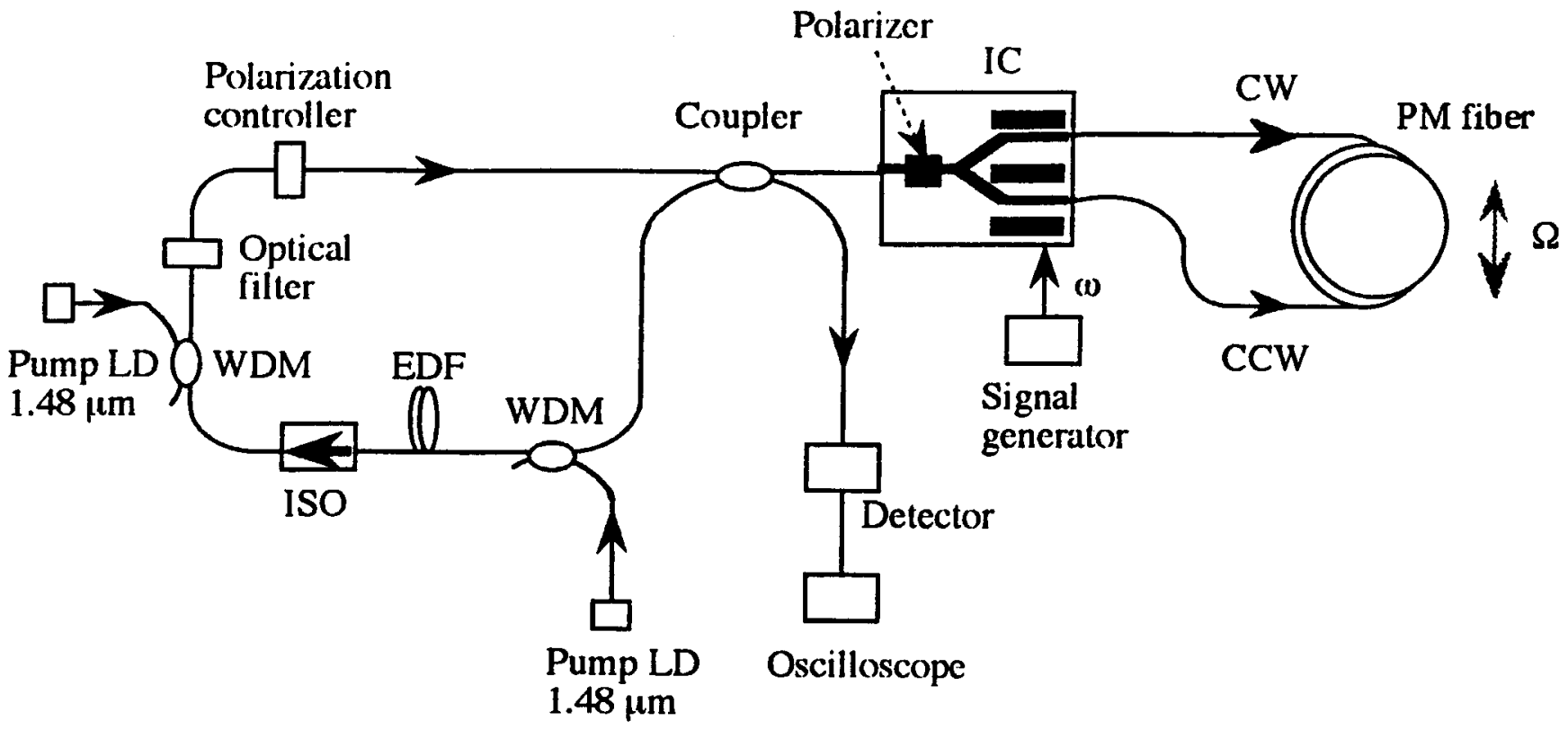

Figure 1 Configuration of our proposed fiber laser rotation sensor and experimental setup 
Er-doped fiber. A piece of Er-doped fiber of $40 \mathrm{~m}$ is oppositely pumped by two $1.48-\mu \mathrm{m}$ LDs through $1.48 / 1.55-\mu \mathrm{m}$ WDM couplers. An isolator is inserted within the feedback loop to ensure the one-direction (clockwise) transmission in the whole system, and improve the performance of fiber amplifier by suppressing the Rayleigh scattering. An optical filter with a 3-nm passband width and a central wavelength of about $1.55 \mu \mathrm{m}$ is placed in the feedback loop. In this experiment a sine-wave electronic signal is applied to the phase modulator of the integrated-optic circuit. The frequency of this electronic phase modulation signal is $f=913 \mathrm{kH}$ (equal to the fundamental cavity frequency), which satisfies the mode-lock condition with the total length (about $227 \mathrm{~m}$ ) of the fiber loop (the average refractive index of fiber is $\mathrm{n}=$ 1.448). In addition, the polarization state is controlled to obtain a maximal system transmission. The output is available from a 3-dB fiber coupler, which also feeds back optical power to the sensing loop through the integrated optic circuit. In the experiment, the end of output fiber to the fast photodetector is carefully cut in a slant plane, so that it will not produce any reflection back to the loop cavity.

\section{EXPERIMENT, SIMPLE ANALYSES, AND DISCUSSION}

The operation of this pulsed fiber laser gyroscope is described as follows. At first, the optical wave generated from the ASE of Er-doped fiber is injected into the sensing loop through the fiber coupler and integrated optic circuit. The lightwave is then separated into two parts by the $\mathrm{LiNbO}_{3}$ integrated optic circuit, in the mean time the opposite sign phase modulation is produced for these two light waves. The separated light waves then propagate over the sensing loop with CW (clockwise) wave and CCW (counterclockwise) wave. Finally, an interfering signal between the $\mathrm{CW}$ and $\mathrm{CCW}$ waves is produced. This produces the intensity modulation due to the time difference and the opposite sign of phase modulation between the $\mathrm{CW}$ and $\mathrm{CCW}$ waves. This interfering signal has the same form as the gyro signal of conventional I-FOG, which is then amplified by the fiber amplifier and fed back to the Sagnac loop through the fiber coupler and integrated optic circuit. In the meantime, some optical power also arrives at the fast photodetector. The feedback light will then continue the procedure mentioned above. Therefore, the total output signal at the fast photodetector is a summation of the interfering signal for each round trip. If the gain of the fiber amplifier is sufficient to compensate for the round-trip losses in the whole system, the laser emission can be produced.

We first look at the case of a CW laser; that is, there is no electrical modulation signal at the phase modulator. Because the optical gain is greater than the round-trip loss, the initial light signal of ASE will get a net amplification each time it travels through the fiber loop. This procedure continues until a strong light signal saturates the gain of fiber amplifier, a balance is reached, and a steady $\mathrm{CW}$ laser emission is then set up. Figure 2 shows the CW laser spectrum, that is, the laser spectrum when the phase modulation signal is not applied (no driven current to the phase modulator). On the other hand, if an electrical signal is placed on the phase modulator and the frequency of phase modulation signal and the length of the whole fiber loop $L$ (including both the sensing loop and feedback loop of Er doped fiber amplifier) satisfies the relation of $\omega \tau=2 i \pi, i=1,2, \ldots(\tau$ is the transmission time of the total round trip, including the time

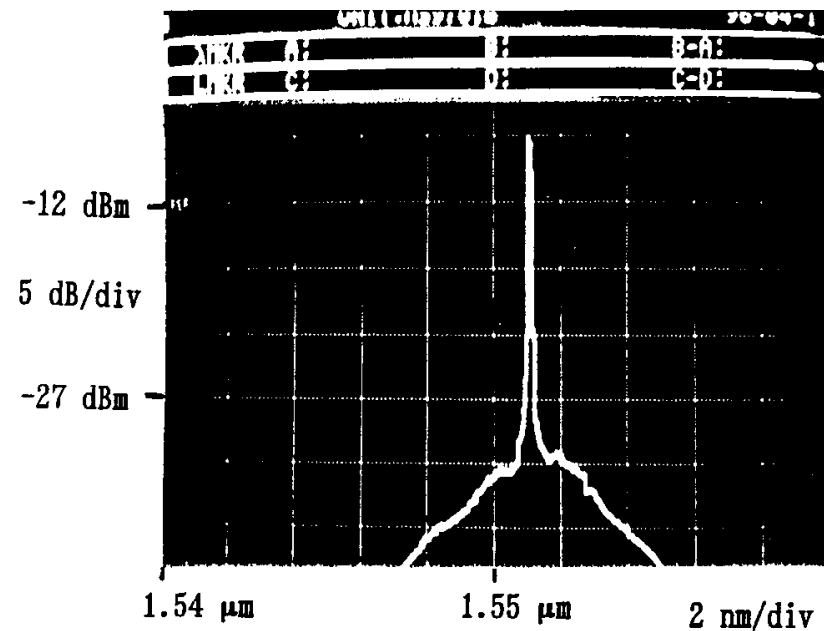

\section{Wavelength ( $\mu \mathrm{m})$}

Figure 2 Optical spectrum of this fiber laser when the modulation signal is not applied (i.e., CW laser operation)

on both the Sagnac loop and Er doped fiber loop), mode lock is generated and the laser emission exhibits a pulse output. Figure 3 is the laser spectrum when the phase modulation signal $(V=9.67 \mathrm{~V})$ is applied and mode locking occurs. The modulation frequency of the phase modulator is $f=913 \mathrm{kH}$, which is just equal to the fundamental cavity frequency. When mode locking occurs, the laser spectrum apparently becomes broader compared with Figure 2. Corresponding to Figure 3, Figure 4 illustrates the measured output pulse of this mode-locked fiber laser. It is seen that the output is of a periodic pulse. The most distinct pulses are obtained when the driven frequency is $913 \mathrm{kH}$, producing two output pulses per modulation period [7]. The double pulse repetition compared with that of the driven signal can be understood from Eq. (1) - the transfer function of the phase-modulated Sagnac interferometer is a cosine function; therefore, if the electronic phase modulation signal is of a sine function, the output of the Sagnac interferometer has two transmission peaks in one period of driven signal. It should also be noted that the pulse duration of our fiber laser is quite long. This is because the modulation frequency of our fiber laser is very small (equal to the cavity frequency of the long fiber resonator), whereas in the homogeneously broadened laser the pulse duration of the actively mode-locked laser depends not only on the atomic linewidth but also on the modulation frequency [8]. The large modulation frequency with the harmonic mode lock would result in the short pulse duration [8], but the harmonic mode lock is not our focus in this article.

In addition to the above discussion, we should point out that when the rotation movement is input into this modelocked fiber laser system, the output pulse of this fiber laser changes because of the Sagnac effect. The rotation rate can be then measured by detecting the peak shift of the output pulse.

We next use a simple method to analyze how the rotation measurement can be performed by this Er-fiber laser gyro system. Based on the above discussions, the first-time interfering signal (i.e., one that has had only one round-trip transmission) arriving at the fast PD has a waveform output 


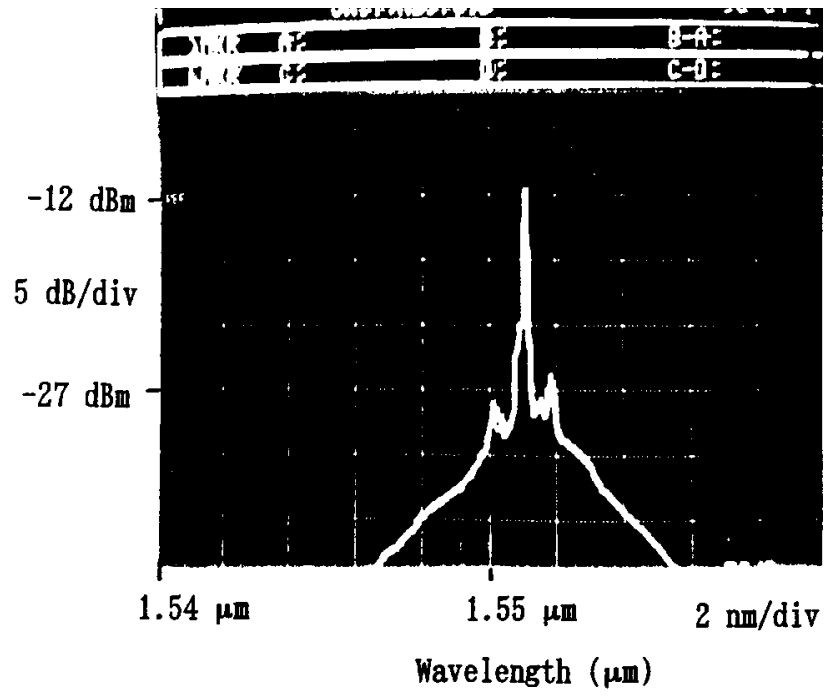

Figure 3 Optical spectrum of the mode-locked fiber laser when the amplitude voltage of modulation signal is $V=9.67 \mathrm{~V}$ and the modulation frequency is just equal to the fundamental cavity frequency $f=913 \mathrm{kH}$

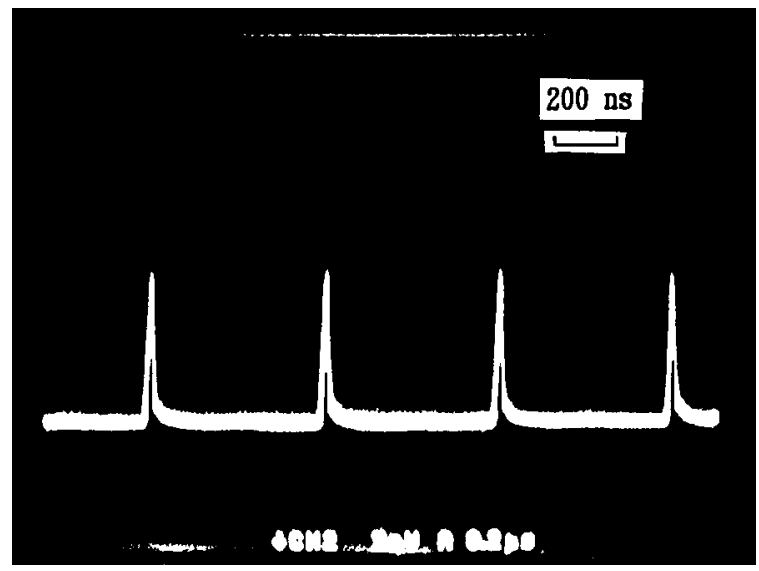

Figure 4 Measured output pulse of the fiber gyro when there is no rotation input (corresponding to Figure 3)

with intensity modulation (the same as the output of Sagnac interferometer) expressed as

$$
P_{1}(t)=K A\left\{1+\nu \cos \left[\phi_{s}+\phi_{e} \cos (\omega t)\right]\right\},
$$

where $\nu$ is a parameter of the fringe contrast, $K$ is a constant reflecting the equivalent round-trip loss (including transmission loss, splicing loss, loss of each optical device, and the splitting loss of fiber coupler), $A$ is the gain of the Er-doped fiber amplifier, and it should be also noted that the gain of optical amplifier is always greater than the round-trip loss in this optical system.

In addition, $\omega$ is the modulation frequency of phase modulation signal $(\omega=2 \pi f), \phi_{e}$ is the effective phase-modulation depth, expressed as

$$
\phi_{e}=2 \phi_{m} \sin \left(\pi f \tau_{s}\right) .
$$

$\tau_{s}$ is the wave-propagation time through the Sagnac loop and is expressed as $\tau_{s}=n l / c, l$ is the length of the Sagnac loop, $n$ is the refractive index of the fiber. $\phi_{s}$ is the Sagnac phase shift induced by the rotation movement, expressed as

$$
\phi_{s}=4 \pi R L \Omega /(\lambda c),
$$

where $\Omega$ is the rotation rate, $R$ the radius of Sagnac loop, $c$ the velocity of light propagation in free space, and $\lambda$ the wavelength of light source.

The above first-time interference optical signal is obtained from the fiber coupler and amplified by the Er-doped fiber amplifier, then fed back into the Sagnac loop through the fiber coupler and integrated optic circuit. Therefore, this interfering signal will then further the propagation described as above. Considering the effect of optical feedback with amplification, the second-time interference signal (i.e., one that has had two round-trip transmissions) has been derived as

$$
\begin{aligned}
P_{2}(t)=A^{2} K^{2}\left\{1+\nu \cos \left[\phi_{s}+\phi_{e} \cos (\omega t-\omega \tau)\right]\right\} \\
\times\left\{1+\nu \cos \left[\phi_{s}+\phi_{e} \cos (\omega t)\right]\right\},
\end{aligned}
$$

and the third-time interference signal (i.e., one that has had three round-trip transmissions) that occurs after feedback has been twice derived is

$$
\begin{aligned}
P_{3}(t)= & A^{3} K^{3}\left\{1+\nu \cos \left[\phi_{s}+\phi_{e} \cos (\omega t-2 \omega \tau)\right]\right\} \\
& \times\left\{1+\nu \cos \left[\phi_{s}+\phi_{e} \cos (\omega t-\omega \tau)\right]\right\} \\
& \times\left\{1+\nu \cos \left[\phi_{s}+\phi_{e} \cos (\omega t)\right]\right\} .
\end{aligned}
$$

The total output at the fast photodetector is the summation of the number of the above-mentioned interfering signals, which is expressed as

$$
P_{\mathrm{tot}}(t)=P_{1}(t)+P_{2}(t)+P_{3}(t)+\cdots,
$$

From Eq.(6), if we let $\omega \tau=2 n \pi, n=1,2,3, \ldots$ (this condition can be realized by proper adjustment of the phase modulation frequency $\omega$ to match the whole round-trip time $\tau)$, in this case the mode lock is generated and the total output signal from the fast photodetector is of a pulse, which can be expressed as

$$
P_{\mathrm{tot}}(t)=\frac{K^{\prime} K A\left\{1+\nu \cos \left[\phi_{s}+\phi_{e} \cos (\omega t)\right]\right\}}{1-K A\left\{1+\nu \cos \left[\phi_{s}+\phi_{e} \cos (\omega t)\right]\right\}},
$$

where $K^{\prime}$ is the photodetecting coefficient.

We next investigate how the rotation can be measured by this fiber gyro system. From the above discussions and Eq. (7) we see that the output is a series of pulses. We can then determine the position of output peaks through the following equation:

$$
P_{\mathrm{tot}}^{\prime}(t)=0 \text {. }
$$

We have

$$
\phi_{s}+\phi_{e} \cos (\omega t)=2 n \pi, \quad n=0,1,2 \ldots .
$$

Equation (9) is a condition required to find the peak positions of the output pulse. From this equation, we see that the peak position depends on the Sagnac phase shift, that is the optical pulse will shift if the rotation occurs. This prop- 


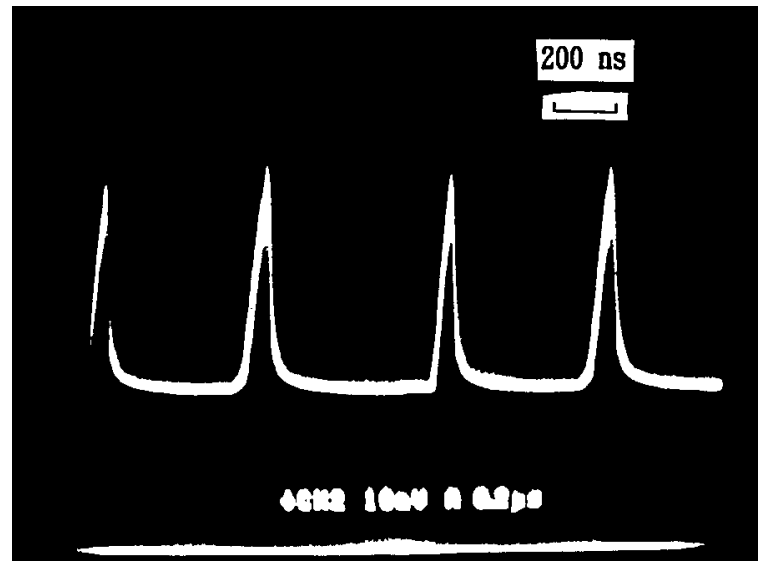

Figure 5 Measured output pulse of the fiber gyro when the input rotation is $\Omega=40 \mathrm{deg} / \mathrm{s}$ (CW rotation) by a mechanical rotation table. The applied voltage of amplitude for the phase modulation signal is $V=3.24 \mathrm{~V}$, and the modulation frequency is $f=913 \mathrm{kH}$

erty can be utilized for the rotation measurement. We first look at the case when there is no rotation input; that is, $\phi_{s}=0$. In Eq. (9), if the effective phase modulation depth is selected to be between $0<\phi_{e}<2 \pi$, Eq. (9) is satisfied only when $n=0$. In this case, the peak positions of the output pulse are determined through the following equation:

$$
\omega t_{0 j}=(2 j+1) \pi / 2, \quad j=0,1,2, \ldots,
$$

where $t_{0 j}$ represents the peak position when there is no rotation input and $j$ denotes the peak number of the output pulse in time axis.

When the rotation occurs, the peaks of output pulse will shift, which are determined by the following equation.

$$
\omega t_{i j}=\arccos \left(-\frac{\phi_{s}}{\phi_{e}}\right)+2 j \pi, \quad j=0,1,2, \ldots,
$$

where $t_{i j}$ represents the peak position when the rotation is input. We then derive the rotation rate from the Eqs. (10) and (11) as

$$
\Omega=\frac{\lambda c}{4 \pi R L} \phi_{e} \sin \omega \Delta t
$$

where $\Delta t$ is the time shift of the output peak due to the rotation, expressed as $\Delta t=t_{i j}-t_{0 j}$, which is a measurable value. We can thus measure the rotation rate by detecting the time shift of the output peak.

Figure 5 demonstrates the output pulse when the clockwise (CW) rotation input ( $\Omega=40 \mathrm{deg} / \mathrm{s}$ ) is supplied through a mechanical rotation table. For a mode-lock operation, the voltage amplitude of modulation signal is $V=3.24 \mathrm{~V}$, and the modulation frequency is equal to the fundamental cavity frequency $f=913 \mathrm{kH}$. We can see that the peak positions of the output pulse are shifted (the separation is changed) when the rotation occurs. In addition, for comparison, in Figure 6 we recorded the output pulse when there is no rotation input (i.e., $\Omega=0$ ). Figure 7 is the measured output when the counterclockwise (CCW) rotation is input $(\Omega=40 \mathrm{deg} / \mathrm{s})$.

Figure 8 shows the calculated result of the time shift of the output peak as a function of the rotation rate with different effective phase-modulation depths. The parameters

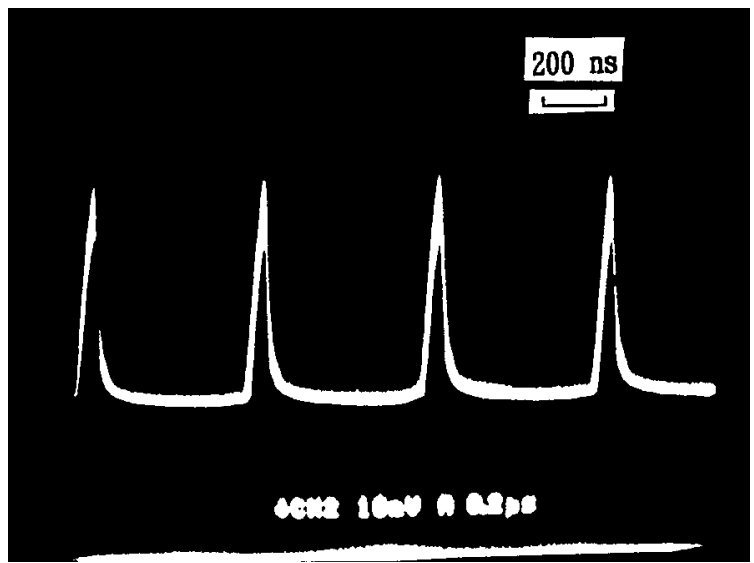

Figure 6 Measured output pulse of the fiber gyro when there is no rotation input. The applied voltage of the amplitude for the phase modulation signal is $V=3.24 \mathrm{~V}$, and the modulation frequency is $f=913 \mathrm{kH}$

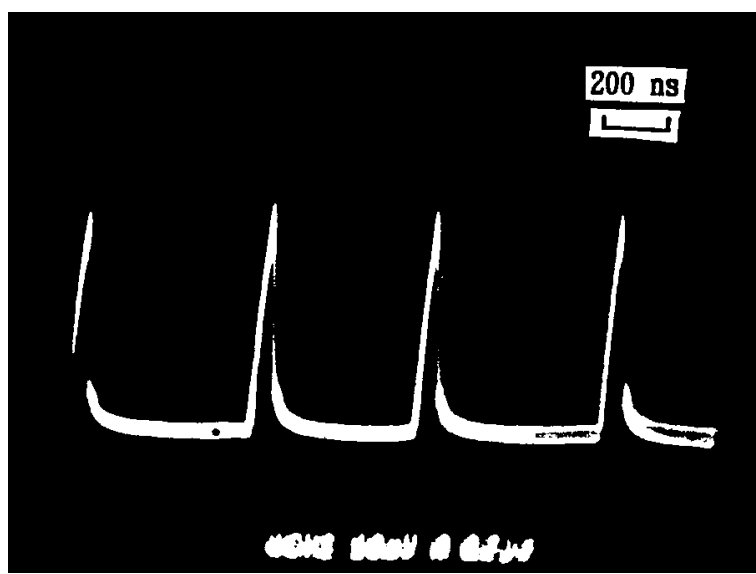

Figure 7 Measured output pulse of the fiber gyro when the input rotation is $\Omega=40 \mathrm{deg} / \mathrm{s}$ (CCW rotation) by a mechanical rotation table. The applied voltage of amplitude for the phase-modulation signal is $V=3.24 \mathrm{~V}$, and the modulation frequency is $f=913 \mathrm{kH}$



Figure 8 Calculated result of the time shift of the output peak as a function of rotation rate with different amplitudes of phase modulation signal

used are directly selected corresponding to the practical values of experiment; that is, $L=227 \mathrm{~m}, f=913 \mathrm{kH}, \lambda=$ $1.55 \mu \mathrm{m}, R=5 \mathrm{~cm}$, and the average refractive index is 


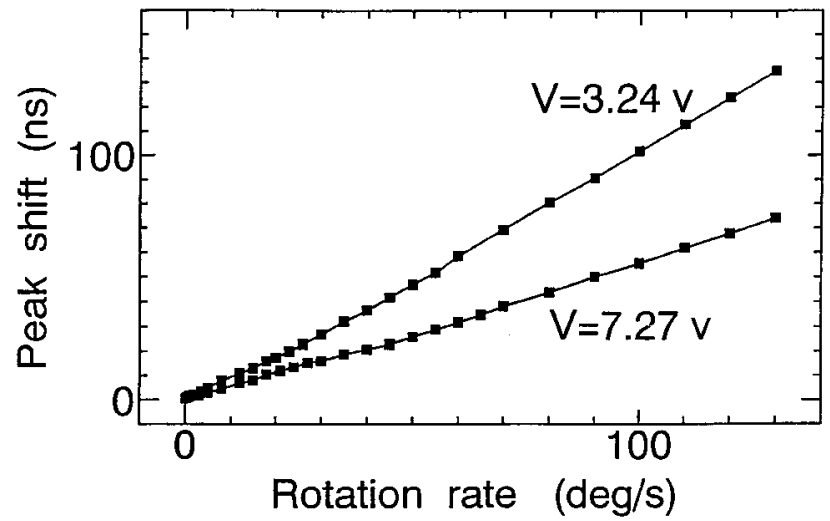

Figure 9 Measured result of the time shift of the output peak as a function of rotation rate with different amplitudes of phase modulation signal

$n=1.447$. From Figure 2, we see that the rotation rate can be derived from the time shift of the output peak. Figure 9 is the measured result of the time shift of the output peak as a function of rotation rate. The rotation rate in the transversal axis corresponds to the practical value input by the mechanical rotation table. From the Figures 8 and 9 it can be seen that the experiment is consistent with the theoretical result. We also see that the slope of the plot is increased when we use a small amplitude voltage of electronic modulation signal (i.e., the phase modulation depth is small), which indicates that the resolution of rotation measurement is increased. However, the small-amplitude modulation signal will broaden the output pulse, and the optical pulse will even disappear [8]. For experimental confirmation, we increased the amplitude of the applied voltage of the electronic phase modulation signal to $V=9.67 \mathrm{~V}$, and kept the same modulation frequency. This can be seen in Figure 4. In addition, Figure 10 also shows the limit case when the amplitude voltage of the phase-modulation signal is small as $V=2.67 \mathrm{~V}$. Comparing Figures 6, 4, and 10, it is apparent that the optical pulse is broadened (this is not favorable) if the amplitude of the phase modulation signal (i.e., phase modulation depth) is reduced. If the amplitude of the phase modulation signal is reduced below $V=2.67 \mathrm{~V}$, the output peak is even disappears. Based on the above discussion, we can now obtain the

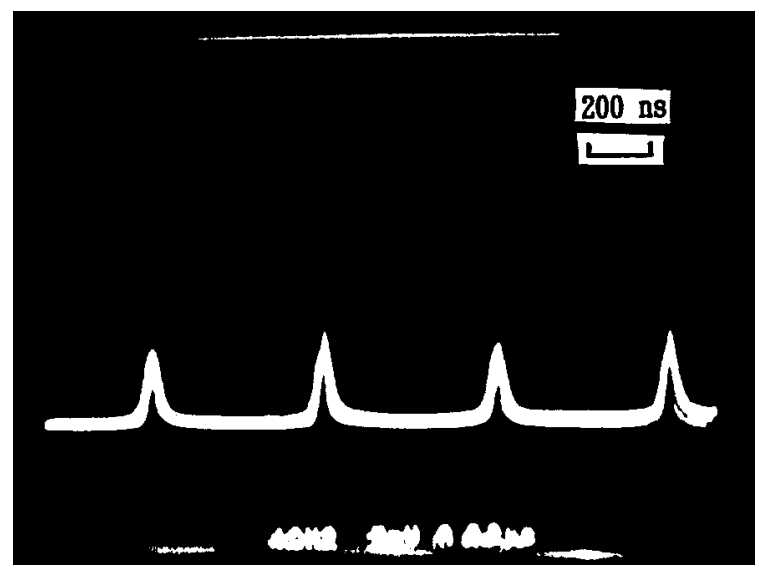

Figure 10 Measured output pulse of the fiber gyro when there is no rotation input. The applied voltage of amplitude for the phase modulation signal is decreased to $V=2.67 \mathrm{~V}$ conclusion that in order to obtain the high resolution of rotation measurement the key point is to use a small phase modulation amplitude of electronic signal and not lose the sharpness of the optical pulse. This can only be realized by reducing the losses (or increasing the optical gain) in the whole optical system. These losses include the insertion loss of each component and the splicing loss. In the present research, the loss of the whole optical system is assumed to be large because of imperfect devices and error operation. We believe that the resolution can be greatly improved if we further reduce the optical loss. Because this optical rotation sensor system works based on the pulsed laser operation, and the rotation measurement can be tested by the peak shift of output pulse. This detection method might be simple in electronics compared with the phase-detection technology of the traditional I-FOG. The whole system is also simple compared with the resonator fiber-optic gyro. Also, the ideas in this article might prove useful for the scientist working on optical-fiber gyros or lasers.

\section{CONCLUSION}

We have proposed a novel pulsed fiber-optic gyro with the use of an integrated-optic circuit, a Sagnac fiber loop, and an Er-doped fiber amplifier. In this optical system, a laser emission in the pulse state was produced because the gain of the fiber amplifier is greater than the round-trip loss and the frequency of phase modulator matched the total loop length (including both the Sagnac loop and the feedback EDFA loop). A simple analysis has been presented and the expression of the output pulse has been derived. When the rotation occurs, the peak of the output pulse will shift. This property has been used for the rotation measurement. It has been experimentally shown that the resolution of rotation measurement is increased if we use a small phase modulation depth; however, the use of a small phase modulation depth will result in the broadening of the output pulse. In order to keep the sharpness of the optical pulse, we should reduce the optical loss (or increase the optical gain) of the whole system.

\section{REFERENCES}

1. H. C. Leferve, "Comments about Fiber-Optic Gyroscope," Proc. SPIE Fiber Opt. Laser Sensors, 1987, pp. 86-97.

2. R. D. Moeller, "Open Loop Output and Scale Factor Stability in a Fiber Optic Gyroscope," IEEE J. Lightwave Technol., Vol. 7, pp. 262-268, 1989.

3. T. Kumagai, H. Kajioka, S. Oho, and H. Snobe, "Development Open-Loop Fiber Optic Gyroscope for Industry and Consumer Use," Proc. SPIE 1785 Fiber Optic and Laser Sensing, Boston, 1992, pp. $74-86$.

4. G. A. Sanders, M. G. Prentiss, and S. Ezekiel, "Passive Ring Resonator Method for Sensitive Intertial Rotation Measurement Geophysical and Relativity," Opt. Lett., Vol. 6, pp. 569-571, 1981.

5. K. Hotate, "Noise Source and Countermeasures in Optical RingResonator Gyro," Proc. OFS'90, Sydney, Australia, 1990, pp. 11-17.

6. S. P. Smith, F. Zarinetchi, and S. Ezekiel, "Stimulated Brillouin Fiber Optic Laser Gyroscope," Opt. Lett., Vol. 16, 1991, pp. 229-231.

7. D. Ostling, P. G. Sinha, and H. Engan, "Spectral Stability and Smoothness of a Phase-Modulated Fiber Laser," Opt. Lett., Vol. 20, 1995, pp. 219-231.

8. A. E. Siegman, Lasers, University Science Books, Mill Valley, CA, 1986.

(c) 1997 John Wiley \& Sons, Inc.

CCC 0895-2477/97 